\title{
Fatigue Evaluation of Offshore Steel Structures Considering Stress Concentration Factor
}

\author{
Oluwafemi John Damilola ${ }^{1}$, Elakpa Ada Augustine², \\ Nwaorgu Obioima Godspower ${ }^{3}$
}

\author{
${ }^{1}$ Department of Mechanical Engineering, ${ }^{2,3}$ Department of Marine Engineering, \\ ${ }^{1,2,3}$ Nigeria Maritime University, Department of Mechanical Engineering, Okerenkoko Delta State, Nigeria
}

Corresponding Author: Oluwafemi John Damilola

\begin{abstract}
The installation of offshore structures and facilities in the marine environment, usually for the production and transmission of oil, gas exploration, electricity, and other natural resources is referred to as offshore construction. Since offshore structures are subjected to changing threats to the environment year-round. Fatigue behavior prediction noticed on these structures should be considered during the design stage. Fatigue is one of the failure mechanisms of offshore steel structures, and it must be investigated properly during system design. The fatigue analysis of offshore structures under drag wave force, total wave force, total moment about the sea bed, and other variables are reviewed thoroughly. The structure's dynamic response becomes a critical aspect in the whole design process. The fatigue analysis was carried out using MATLAB software, material properties of the offshore structure, and wave spectrum characteristics in this study. This study shows the JONSWAP spectrum and stress concentration analysis prediction. The offshore support structure that is predicted during the design phase will help to keep the stress concentration factor below the fatigue threshold and anticipate safe life design, according to the results of the fatigue study. The fatigue performances of tripod and jacket steel support structures in intermediate waters depth are compared in this project $(20-50 \mathrm{~m})$. The North Atlantic Ocean is used as a reference site, with a sea depth of 45 meters. The tripod and jacket support structures will be designed by using current industry standards.
\end{abstract}

Keywords: [Fatigue evaluation, North Atlantic Ocean and Failure]

\section{INTRODUCTION}

Offshore buildings are primarily utilized in the petroleum sector for drilling, extraction, storage of crude oil and natural gas. Phillips Petroleum erected the first oil platform in the North Sea in Grand Lake St. Mary's, Ohio, in 1891. Fatigue is one of the failure modes of offshore steel structures, and it must be thoroughly investigated during the design phase. Offshore structures, unlike land structures, are influenced by the ocean environment (Agarwal et al. 2011).

Other environmental loads, such as current and wave, are applied to the structure at the same time as the wind load. The majority of these complex loads are borne by support structures. Because the parameters of the installation location have such a large influence on maritime composite loads, load prediction and evaluation is a critical task before design. Furthermore, the support structure accounts for a significant portion of the overall system cost, and the installation cost is excessive (Duncan, 2005).

As a result, ocean environmental impact assessment is a vital procedure for cost-effective design. Furthermore, because the structures are exposed to the sea environment for the duration of their design life, the constantly operating loads have a substantial impact on the structure's lifetime. 
Typhoons, for example, can cause a loss of function or structural collapse (API 2007). Even if a relatively minor composite load is applied constantly, fissures in the weak member may form, leading to collapse. Fatigue is the term for the damage caused by a modest but constant load operating on a structure. Design methods for fatigue damage of offshore wind turbine support structures are presented in various design criteria (DNV 2011).

In addition, studies on fatigue damage have been performed variously by many researchers. Those are evaluation of fatigue damage in the time domain and frequency domain and fatigue reliability evaluation. These studies estimate the probability distribution of loads from the observed data during relatively short period of time compared with design life and assume the observed data will be repeated for the future (Dong et al. 2011).

In another study, fatigue damage was calculated by defining the stress transfer function in the frequency domain. However, the distribution of loads has a different shape every year (Yeter et al. 2015). For example, the wind speed in the next year may be higher or lower than the observed year. The assumption that the same wind speed will be repeated in the future is not reasonable and might give bad estimation of fatigue life of the structure (Dong et al. 2012).

The purpose of this study is to investigate how the variability in ocean environment affects the offshore structure. Simulation was performed by using S-N curve to estimate the probability distribution of loads. Through MATLAB, the wave kinematics and the computation of the various forces which includes the computation of the inertia and drag forces is calculated using the Morison equations. Fatigue damage was calculated using JONSWAP spectrum. The dynamic behaviour of structures becomes very important parameter in the overall design procedures. An attempt at solving the fatigue problem of a typical offshore support platform by applying those formulations through the development of a MATLAB computer routine code is also carried out.

\subsection{METHODOLOGY}

The total wave forces on an offshore structure was postulated by Morrison amongst others, which shows that the wave force on an offshore structure is in form of inertia force and drag force. Morrison also stated the condition for the equation to hold while assuming a cylinder case for it experiment (Morrison et al, 1950). According to Sarpkaya \& Issacson, (1981) to calculate the wave load on an offshore structure, there is need to know the wave parameters like the wave maximum height, wave length, wave period, wave frequency and wave number, also the water parameters like water depth, water density and water viscosity.

\subsection{Wave length}

Wave length is a measure of distance between two identical peaks that is high points or low points in a wave.

$\lambda_{0}=\frac{g}{2 \times \pi} \times T^{2}$

$\lambda_{0}=$ The water wave length at deep sea

$g=$ The acceleration due to gravity

$T=$ The wave period

\subsubsection{Wave number}

The wave number is the spatial frequency of a wave either in cycles per unit wave length or radians per unit wave length. $k=\frac{2 \times \pi}{\lambda}$

$\mathrm{k}=$ The wave number

\subsubsection{Wave frequency}

The wave frequency describes the number of waves that pass the fixed offshore structures place at the sea in a given amount of time. $w=\sqrt{g \times k \times \tanh (k \times d)}$

$\mathrm{w}=$ The wave angular frequency

Calculate time $t$ 

factor.

$t=\sqrt{\frac{2 \times \pi \times \lambda}{g}}$

\subsection{Drag wave force}

The wave drag force is defined by the film of water wave which is a function of the flow velocity, water density and dynamic viscosity.

$F_{d}=\frac{D \times \pi \times \rho \times H_{\max }{ }^{2} \times \lambda}{T^{2}} \times\left[\left(A_{2} \times C d \times|\cos \theta| \times \cos \theta\right)\right]$

$F_{d}=$ The drag wave force

\subsubsection{Total wave force}

The total wave force is the sum of the inertia force and drag force at different wave angle, this shown the entire force imparted on the offshore structure by the wave at different wave angle under linear water flow.

$F_{T}=F_{m}+F_{d}$

$=\frac{D \times \pi \times \rho \times H_{\max }{ }^{2} \times \lambda}{T^{2}} \times\left[A_{1} \times C m \times \sin \theta+\right.$

$\left.\left(A_{2} \times C d \times|\cos \theta| \times \cos \theta\right)\right]$

$F_{T}$ The total wave force

\subsection{Total moment about the seabed}

$M=\frac{D \times \pi \times \rho \times H_{\max }{ }^{2} \times \lambda^{2}}{T^{2}} \times\left[A_{3} \times C m \times\right.$

$\left.\sin \theta+\left(A_{4} \times C d \times|\cos \theta| \times \cos \theta\right)\right]$

(7)

$M=$ The total moment about the seabed

Note $\theta$ move from $0^{0}$ by a step of $10^{0}$ which can be change by the program user, to get the different wave force and moment about seabed at different value of $\theta$. A plot of different value of against the corresponding value of Total force and Moment about the seabed is considered.

\subsection{Stresses on each Member of the Offshore Structure}

The stress on each member can be calculated using the general formula for stress calculation

$\sigma_{m}=\frac{E}{L} \times\left\{\begin{array}{llllll}C_{x} & C_{y} & C_{z} & -C_{x} & -C_{y} & -C_{z}\end{array}\right\} \times\left\{\begin{array}{c}\dot{x}_{n} \\ \dot{y}_{n} \\ \dot{z}_{n} \\ \dot{x}_{n} \\ \dot{y}_{n} \\ \dot{z}_{n}\end{array}\right\}$

$\sigma_{m}$ Stress of each member
The solution should use the scalar multiplication approach to resolve as shown below for each member stress

$$
\begin{aligned}
& \sigma_{\mathrm{m}}=\frac{\mathrm{E}}{\mathrm{L}} \times\left[\left(\mathrm{C}_{\mathrm{x}} \times \dot{\mathrm{x}}_{\mathrm{n}}\right)+\left(\mathrm{C}_{\mathrm{y}} \times \dot{\mathrm{y}}_{\mathrm{n}}\right)+\right. \\
& \left(\mathrm{C}_{\mathrm{z}} \times \dot{\mathrm{z}}_{\mathrm{n}}\right)+\left(-\mathrm{C}_{\mathrm{x}} \times \dot{\mathrm{x}}_{\mathrm{n}}\right)+\left(-\mathrm{C}_{\mathrm{y}} \times \dot{\mathrm{y}}_{\mathrm{n}}\right)+ \\
& \left.\left(\mathrm{C}_{\mathrm{z}} \times \dot{\mathrm{z}}_{\mathrm{n}}\right)\right]
\end{aligned}
$$

\subsubsection{Stress concentration factor}

The stress concentration factor can be estimated based on the parameters and formulas established as shown in fig 1 . Depending on different configuration of the joint, the stress concentration varies from one form to the other. For atypical chord saddle, the stress concentration factor is given as

$S C F=\gamma \tau^{1.1}\left[1.11-3(\beta-0.52)^{2}\right] \sin \theta^{1.6}$

Where

$\gamma=\frac{D}{2 T}$

$\tau=\frac{t}{T}$

$\beta=\frac{b}{D}$

Where $\mathrm{D}=$ diameter of the cylindrical support, $\mathrm{t}=$ thickness

The typical joint bracing configuration of the saddle is shown below

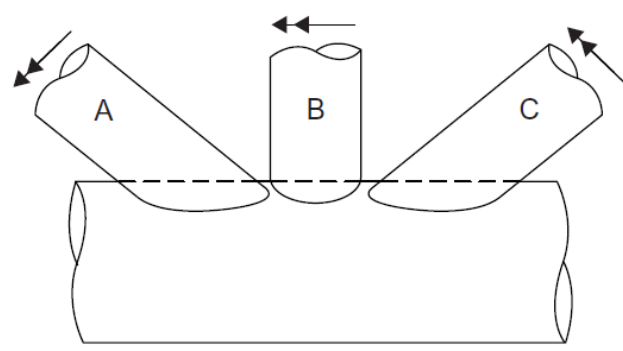

Fig 1: Typical bracing configuration of an offshore support joint

\subsubsection{Fatigue analysis}

The Fatigue damage analysis is carried out within the framework of the Miner Palmgren theory (Sutherland 1999). Denoting by $\mathrm{N}$ the maximum number of stress cycles that a material can withstand under a stress range $S$, it yields

$N=k-S-m \quad(11)$

The S-N curve generated can be used in estimating the damage life and 

factor.

lifespan of the structure. The basic S-N Where curve is of the form

$\log _{10}(N)=\log _{10}\left(k_{1}\right)+m \log _{10}(s)$

$\mathrm{N}=$ predicted number of cycles to failure under stress range $\mathrm{s}$

$\mathrm{K} 1=$ material constant.

Typical S-N curves are shown below

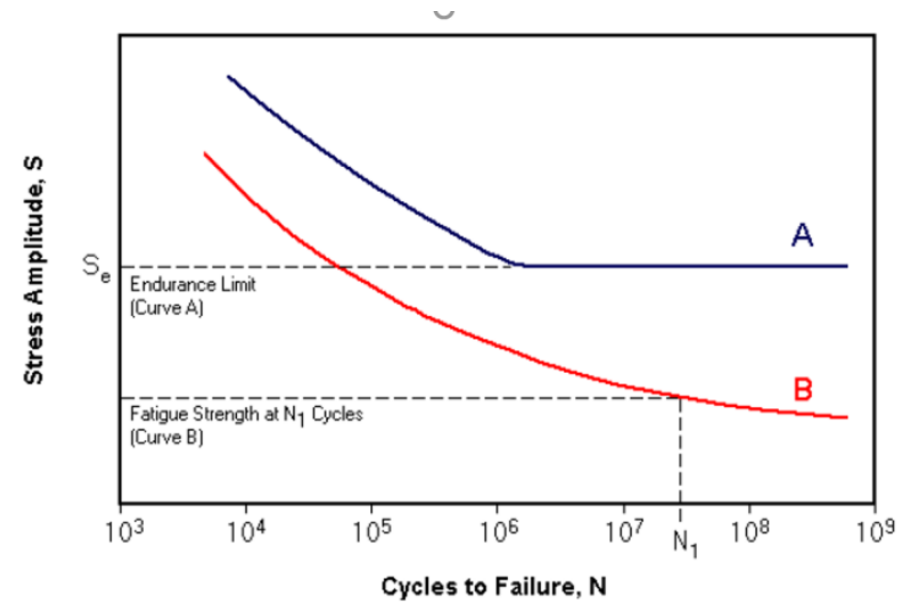

Fig 2: Typical S-N curve

Where $m$ is the Wöhler integer exponent depending on the material and $\mathrm{k}$ is a structure depending parameter. For constructional steel, $m=3$ is generally set.

\subsection{Deterministic Spectral analysis}

The spectrum used for this analysis is the JONSWAP spectrum. Although any spectrum can be used but for the purpose of this analyses this was chosen. The general expression for this energy spectrum is given as

$s_{\eta \eta(f)}=\frac{\beta g^{2}}{2 \pi^{4} f^{5}} \exp \left(-\frac{5}{4}\left(\frac{f_{p}}{f}\right)^{4}\right) \gamma^{a}$

Where

$\gamma=$ peak en Dancment factor, $1<\gamma<7$

$\beta=603.9\left(\frac{H_{S} f_{p}^{2}}{g}\right)^{2.036(1-0.298 \ln (\gamma)}(14)$

The expression for gamma is

$\gamma=5.55\left(174\left({ }^{H_{s}} / g T_{z}^{2}\right)\right)^{\frac{1}{4}}$

The first moment $m_{o}$ as

$m_{o}=\int_{0}^{\infty} s_{\eta \eta}(f) d f$

The second moment of the spectrum

$\left.m_{2}=\int_{0}^{\infty} s_{\eta \eta}(f) f^{2} d f 17\right)$

The significant stress

$\sigma_{s}=4 m_{o}^{0.5}$
The upcrossing period

$T_{z z}=\sqrt{\frac{m_{o}}{m_{2}}}$

The cycle to stress in a given time, $t$ is given as

$n=\frac{3600 * 24 * 356 * t}{T_{z z}}(20)$

Hence the damage and fatigue life can then be computed as

$D=\frac{n}{N}$

fat $_{\text {life }}=1 / D$

Where $\mathrm{N}$ can be gotten from the S-N curve for the respective material.

\subsection{RESULTS AND DISCUSSION}

The characteristic structure features, including its geometrical properties, as well as the wave spectrum parameters employed in this research, will be detailed. The impact of forces on structures, stress concentration variables, and damage life will all be shown and briefly reviewed here.

\subsection{Specification of the Jacket platform support}

The following are the properties of the support structure used for this analysis which is made of steel material. 

factor.

Table 1: Showing material property of an offshore support (Rohith, 2017)

\begin{tabular}{|l|l|}
\hline Material & Properties/values \\
\hline Young's modulus & $2.1 \times 108 \mathrm{kN} / \mathrm{m}^{2}$ \\
\hline Density of steel & $7.85 \times 10^{3} \mathrm{~kg} / \mathrm{m}^{3}$ \\
\hline Density of sea water & $1.025 \times 10^{3} \mathrm{~kg} / \mathrm{m}^{3}$ \\
\hline Hydrodynamic inertia coefficient, CM & 2.0 \\
\hline Drag coefficient & 0.6 \\
\hline Acceleration due to gravity, g & $9.81 \mathrm{~m} / \mathrm{sec}^{2}$ \\
\hline Diameter of the pile & $1.2 \mathrm{~m}$ \\
\hline Diameter of the horizontal bracing & 0.65 \\
\hline Diameter of transverse bracing & 0.65 \\
\hline
\end{tabular}

The sea spectrum used is the JONSWAP spectrum with the following properties

\begin{tabular}{|l|l|}
\hline Properties & Values \\
\hline Significant wave height h_s & $7 \mathrm{~m}$ \\
\hline Wave steepness & $1 / 20$ \\
\hline Frequency range & $0.02-0.4 \mathrm{HZ}$ \\
\hline
\end{tabular}

The JONSWAP spectral energy density spectra with a $2 \mathrm{~Hz}$ up crossing frequency were used in this investigation. The energy density corresponding to the given frequency is generated by this energy spectrum. The energy density should rise as the frequency rises, as expected. In the frequency range of 0 to $0.42 \mathrm{~Hz}$, this was evident. As the frequency is increased, the energy density drops sharply from 0.42 to $1.2 \mathrm{~Hz}$, as illustrated in fig 3. From 1 to 1.2 wave frequency, the energy spectrum tends to zero. The Raleigh ray frequency curve is depicted by the spectral energy density curve.

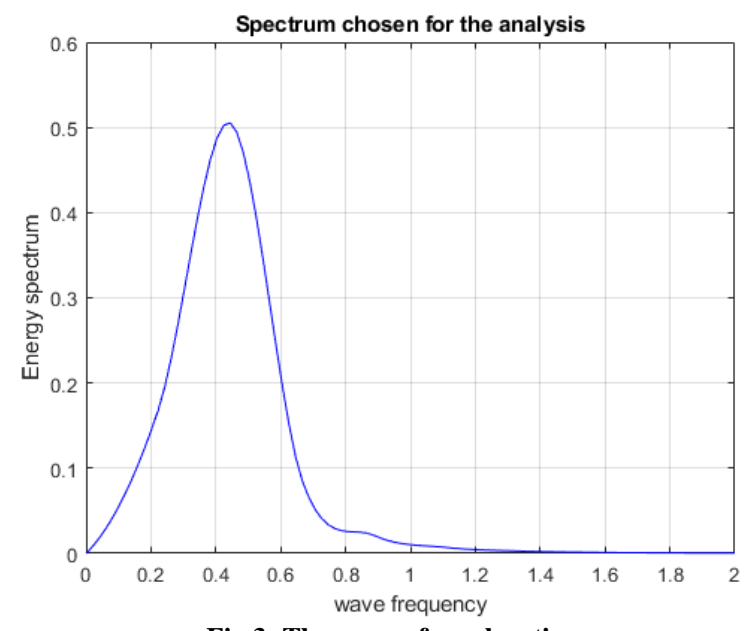

Fig 3: The sea surface elevation

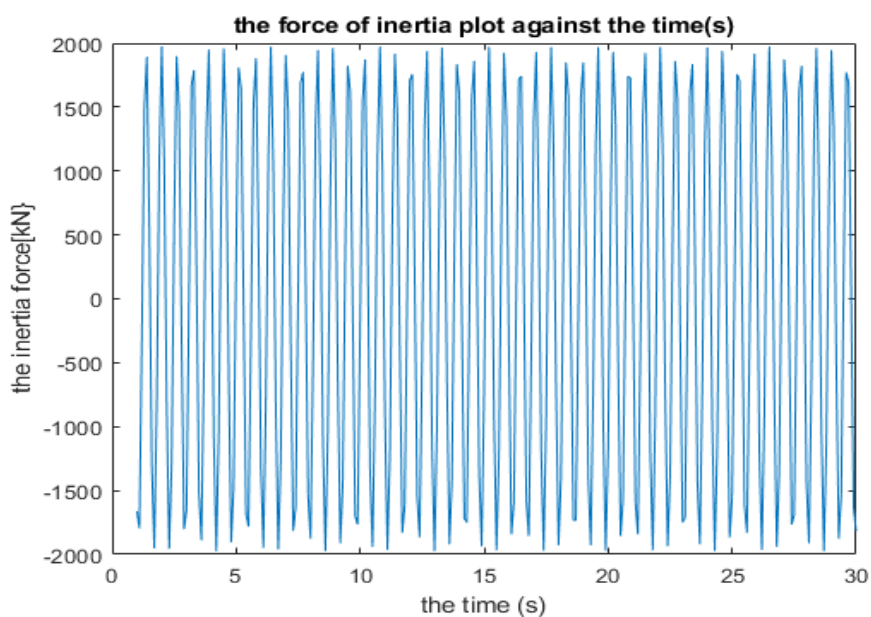

Fig 4: plot of the Inertia force exerted on the structure by the spectrum.

The inertia force was calculated due to the presence of the wave interacting with the offshore structure. This was necessary due to the anticipated estimation of the requisite stress required for the fatigue prediction computation. As demonstrated in figs 4 to 6 , an inertial force resists a change in the FPSO's time. The vibration phase of the offshore structure can easily be seen in Figs 4 and 5. Fig 4 demonstrates how the 

factor.

momentum of water changed over time, implying that the force of $2000 \mathrm{KN}$ exerted by water flow on the support structure changes with time. The resistance and total force of the support structure created by the motion of the water vary with time, as shown in Fig5. The spectrum produces a total force of $6^{*} 10^{5} \mathrm{kN}$, according to the simulation result shown.

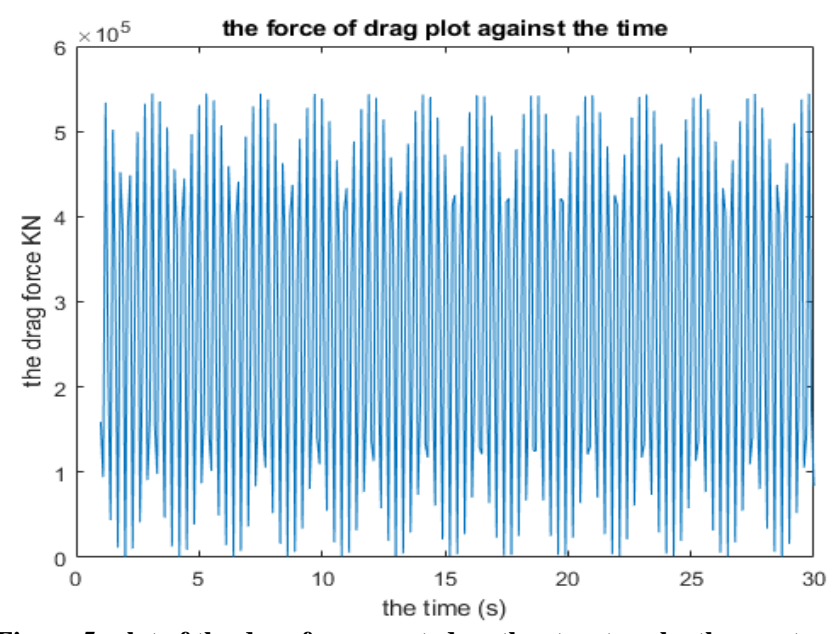

Figure 5: plot of the drag force exerted on the structure by the spectrum.

\subsection{CONCLUSION}

The fatigue analysis of an offshore support system was carried out in this study. It began with a consideration of the theoretical formulation of the forces acting on the structures, wave kinematics, and the computation of the various forces, which included the computation of inertia and drag forces using the Morison equations. The S$\mathrm{N}$ diagram, which is also a regular technique in the industry, was used, as well as additional fatigue analysis calculations.

A spectral analysis approach that integrates the application of the sea surface elevation was also attempted. This is done by factoring in the sea spectrum, and the JONSWAP spectrum was picked for this study among a variety of sea spectra ranging from Ochi to the Pierscomosciwitz spectrum (PM). This was because it added value to actual implementations and the outcomes obtained from this sea state through time have proven to be practicable.

The computation is also carried out using MATLAB (Matrix Laboratory) source code. This language is chosen because it is the technical language for scientific computations, and it includes all of the necessary written functions and libraries for engineering calculations. Unlike other programming languages such as Java or Python, most of these are general-purpose languages that aren't specifically designed for engineering calculations.

Other fatigue analytical methods are, however available and results when compared to spectral analysis which reveal a few inconsistencies. This is always done with the use of some factor, such as constant multipliers which can be justified by the structure's parameter. Standard computer methods, such as SACS, have been used by offshore installation managers (OIM) and designers for fatigue and other structural behavioral assessments of offshore support systems for a long time. This research work details the theoretical and mathematical formulations of the forces acting on the offshore support platform that in turns causes the cyclic loading on the elements of the structure. Also, it attempts at solving the fatigue problem of a typical offshore support platform by applying those formulations through the development of a MATLAB computer routine code.

Acknowledgement: None 


\section{Conflict of Interest: None}

\section{Source of Funding: None}

\section{REFERENCES}

1. Agarwal, P. and Manuel, L. (2011), "Incorporating irregular nonlinear waves in coupled simulation and reliability studies of offshore wind turbines", Applied Ocean Research, 33(3), 215-227.

2. AISC (2005), Specification for structural steel buildings, AISC 360-05.

3. API, RP 2A-WSD (2007), Recommended Practice for Planning, Designing and Constructing Fixed Offshore PlatformsLoad and Resistance Factor Design, Washington DC, USA: First Edition, American Petroleum Institute.

4. DNV (2011), Fatigue design of offshore steel structures, Recommended Practice DNVRP-C203.

5. Dong, W., Moan, T. and Gao, Z. (2011), "Long-term fatigue analysis of multi-planar tubular joints for jacket-type offshore wind turbine in time domain", Engineering Structures, 33(6), 2002-2014.
6. Duncan, Cynthia. (2005). The 2005 AISC Specification for Structural Steel Buildings: An Introduction. 1-5. 10.1061/40753(171)162.

7. Morison, J.R., Johnson, J.W., and Schaaf, S.A. (1950), "The force exerted by surface waves on piles", Journal of Petroleum Technology, 2(5), 149-154.

8. Rohith.T, and Jayalekshmi. R (2017). Deterministic and Spectral Fatigue Analysis of Tubular Joints of a Jacket Platform. International Journal of Scientific \& Engineering Research 8(11): 149

9. Sarpkaya T and Isaacson M, 1981, Mechanics of Wave Forces on Offshore Structures, Van Nostrand Reinhold.

10. Sutherland, Herbert J. 1999. On the Fatigue Analysis of Wind Turbines. Albuquerque, New Mexico: Sandia National Laboratories.

How to cite this article: Damilola OJ, Augustine EA, Godspower NO. Fatigue evaluation of offshore steel structures considering stress concentration factor. International Journal of Research and Review. 2021; 8(10): 307-313. DOI: https://doi.org/10.52403/ijrr.20211041 